\title{
Reconstruction of temporal hollowing using two alloplastic materials simultaneously with titanium mesh and a silicone implant
}

\author{
Han Byeol Jin, Jee Hyeok Chung, \\ Kyung Sik Kim, Seung Hong Kim, \\ Joon Choe, Jeong Yeol Yang
}

Department of Plastic and Reconstructive Surgery, Myongii Hospital, Goyang, Korea
Temporal hollowing is a contour deformity that results in a concavity or hollowing of the temporal region, causing significant cosmetic problems that affect patients both physically and psychologically. For these patients, cranioplasty is needed for protective coverage of the brain and to restore a pleasing aesthetic contour to the cranium. We report a case in which titanium mesh was used as a customized craniofacial implant for a bony defect and a silicone implant was used for soft tissue augmentation of muscle and to address temporal fat pad atrophy. The procedure resulted in high patient satisfaction from an aesthetic standpoint and, importantly, restored a functional barrier resistant to trauma.

Keywords Temporal bone / Titanium / Silicone

\section{INTRODUCTION}

Temporal hollowing refers to a depression in the temporal region, including the lateral orbital rim and zygomatic arch [1]. This deformity may follow zygomaticotemporal fractures, surgical coronal incisions, and lipopenia resulting from certain diseases. The pathophysiology of temporal hollowing involves atrophy of the superficial temporal fat pad caused by ischemia, displacement, or denervation [2,3]. Hollowing defects cause significant cosmetic problems that affect patients both physically and psychologically [4]. For these patients, cranioplasty is needed to provide protective coverage for the brain and to restore a pleasing aesthetic contour to the cranium. Herein, we report a case of temporal hollowing with a bony and soft tissue defect that was reconstructed using titanium mesh and a silicone implant.

Received: Aug 30, 2018 Revised: Sep 19, 2018 Accepted: Sep 25, 2018 Correspondence: Jee Hyeok Chung Department of Plastic and Reconstructive Surgery, Myongji Hospital, 55 Hwasu-ro 14beon-gil, Deokyang-gu, Goyang 10475, Korea

Tel: +82-31-810-6830, Fax: +82-31-810-6837, E-mail: gabriel@mjh.or.kr

Copyright @ 2019 The Korean Society for Aesthetic Plastic Surgery.

This is an Open Access article distributed under the terms of the Creative Commons Attribution Non-Commercial License (http://creativecommons.org/licenses/by-nc/4.0/) which permits unrestricted non-commercial use, distribution, and reproduction in any medium, provided the original work is properly cited. $\quad w w w . e-a a p s . o r g$

\section{CASE REPORT}

A 23-year-old man visited plastic surgery department of Myongji Hospital with a chief complaint of temporal hollowing and requested augmentation surgery to correct the defect to match the contralateral side. The patient underwent a craniectomy for a subdural hemorrhage at our hospital 2 years prior following a pedestrian traffic accident. Thereafter, a surgical site infection occurred and tensor fascia lata duraplasty was performed 1 month after the craniectomy. The patient underwent cranioplasty 4 months after duraplasty using banked autologous cranial bone. On physical examination, pulsation of the brain parenchyma was grossly palpable in the central portion of the bony defect in the temporal hollowing region, and this area required adequate protection. The patient presented with facial nerve palsy of the temporal branch in addition to severe temporal hollowing. On palpation, the soft tissue was sufficiently thin to allow the bone to be felt just below the overall temporal hollowing site. We performed 3-dimensional (3D) computed tomography (CT) in order to comprehensively identify the extent of the defect in the temporal area of the patient. A significant amount of bone had been absorbed in the area where cranioplasty was performed using autologous cranial bone. The bony defect area of the cranioplasty site was measured as $108.9 \times 77.0 \mathrm{~mm}$, and the temporalis muscle was only present to a minimal extent due to severe atrophy that was speculated to have resulted from the 
denervated, devascularized state of the temporal area caused by surgical site infection. Consequently, the patient exhibited severe left frontotemporal hollowing caused by a combination of temporal bone defect, muscle atrophy, and atrophy of the superficial temporal fat pad (Fig. 1). The patient declined a bone graft due to concerns about donor site morbidity, and instead requested reconstruction using an implant with strength comparable to that of bone. The author made a request to a 3D implant printing company (JEIL Medical Corp., Seoul, Korea) to manufacture a prefabricated titanium mesh. The manufacturer virtually mirrored the skull and bony defects using CT scan data and prepared a mesh with banding and preconditioning matching the skull contour (Fig. 2). The programs used for 3D reconstruction were InVesalius and SolidWorks. For augmentation of the soft tissue, a silicone implant was prepared in block form and was carved and inserted during surgery, with coverage by the skin flap to simulate the postoperative results. This patient had almost no residual temporalis muscle and also had facial nerve palsy, therefore, there was no alternative to alloplastic material for soft tissue augmentation.

Surgery was performed under general anesthesia. A unicoronal incision was made and a frontoparietotemporal skin flap was elevated through the subperiosteal layer until the hollowing area was fully exposed. In the bony defect area, the skin was adhered to the dura and was meticulously dissected by a neurosurgeon. The metal plates and screws that were fixed from a previous surgical intervention were all removed. The prefabricated titanium mesh was placed over the bony defect and was fixed in place with screws. A silicone block was carved to match the frontotemporal contour of the contralateral side and was fixed using a nylon suture (Fig. 3). A Hemovac drain was also inserted around the silicone implant. At the same time, the author performed excision of the hypertrophic scar from the incision at the time of the previous operation and finished the operation with tension-free layer-by-layer suturing. After surgery, the patient was instructed to take great care to maintain an appropriate position to reduce the likelihood of implant displacement and seroma formation. The hemovac was removed 4 days after surgery, and stitch removal was performed 7 days after surgery. The patient

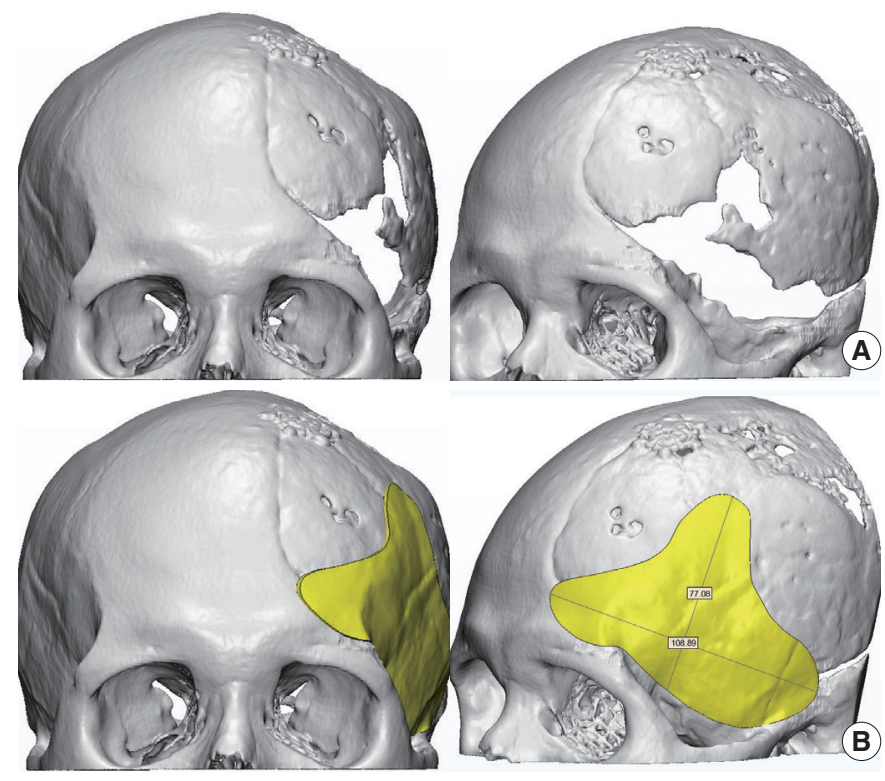

Fig. 2. Three-dimensional (3D) model of the prefabricated titanium mesh (JEIL Medical Corp). The manufacturer virtually mirrored the skull and bony defects using computed tomography scan data (A) and prepared a mesh with banding and preconditioning matching the skull contour $(B)$. The programs used for 3D reconstruction were InVesalius and SolidWorks.
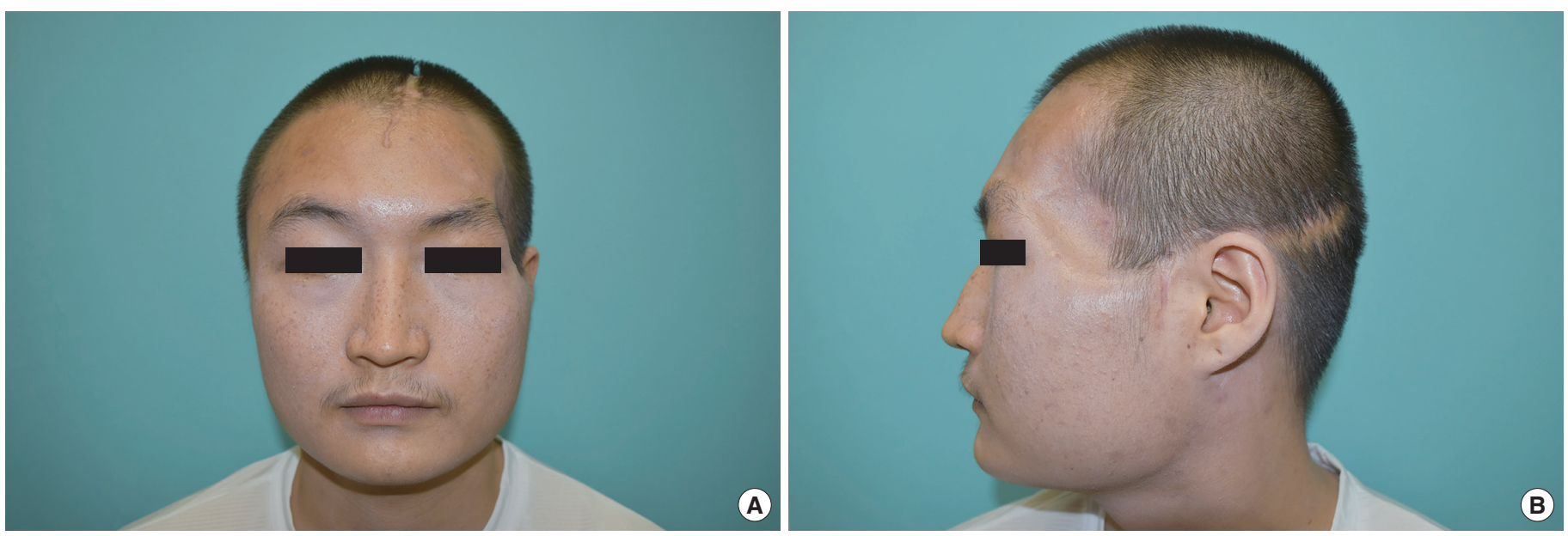

Fig. 1. Preoperative photographs. (A, B) Note the severe left frontotemporal hollowing and deformity caused by a combination of temporal bone defect, muscle atrophy, and atrophy of the superficial temporal fat pad. Pulsation of the brain parenchyma was grossly palpable in the central portion of the bony defect in the temporal hollowing region, and this area required adequate protection. The patient presented with facial nerve palsy of the temporal branch in addition to severe temporal hollowing. 

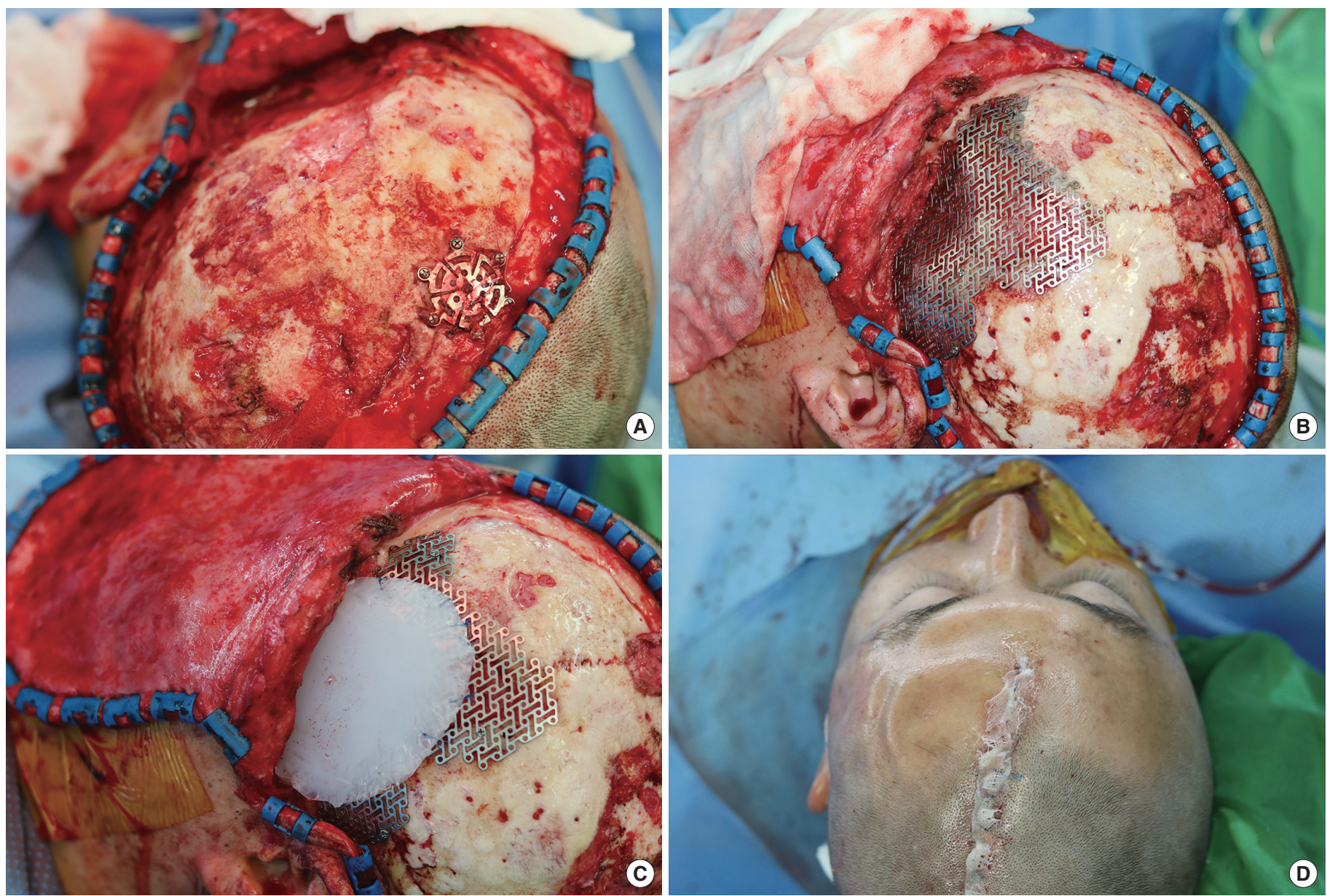

Fig. 3. Intraoperative photographs. (A) Dissection of the skin flap from the dura. (B) Inset and fixation of the prefabricated titanium mesh on the bony defect area. (C) Placement of the silicone block on the soft tissue defect area. (D) Immediate postoperative bird's eye view showed symmetrical temporal area.

recovered with no complications such as surgical site infection, seroma formation, hematoma formation, implant displacement, or extrusion. The patient was functionally and cosmetically satisfied with the result and has received follow-up for 3 months at the outpatient clinic. As of the most recent visit, no implant displacement, seroma formation, or wound infection had occurred (Fig. 4). The author plans to continue long-term follow-up of this patient.

\section{DISCUSSION}

Temporal hollowing refers to a temporal contour deformity that produces a hollowing of the temporal region. It results from surgical coronal incision, primary trauma, progressive hemifacial atrophy, craniofacial microsomia, and other diseases [3]. The most common cause of temporal hollowing is a surgical coronal scalp incision leading to atrophy of the superficial temporal fat pad caused by ischemia, displacement, or denervation [3]. Magnetic resonance imaging studies of patients presenting with temporal hollowing have identified decreased volume or prolapse of the superficial temporal fat pad [1]. Kim and Matic [3] identified a fibrous network running between the leaves of the deep temporal fascia that potentially provides support for the superficial temporal fat pad. This suspensory system is extremely adherent superficially and any attempt to elevate the superficial fascia from the fat pad would inevitably result in disturbing the fat pad itself, disrupting the blood supply to the entire lobule [3]. Fat distribution and physiology is in part controlled by the autonomic nervous system. The zygomaticotemporal nerve travels between the superficial and deep layers of the deep temporal fascia [3]. If the zygomaticotemporal nerve, which is part of the trigeminal ganglion, is injured during surgery, retrograde signaling from the injury to the zygomaticotemporal nerve could trigger an autonomic response from the trigeminal ganglion, causing metabolic changes and atrophy of the superficial temporal fat pad [3]. In this patient, the scar from the incision made during previous surgery was located in an area that could lead to injury of zygomaticotemporal nerve, and the su- 

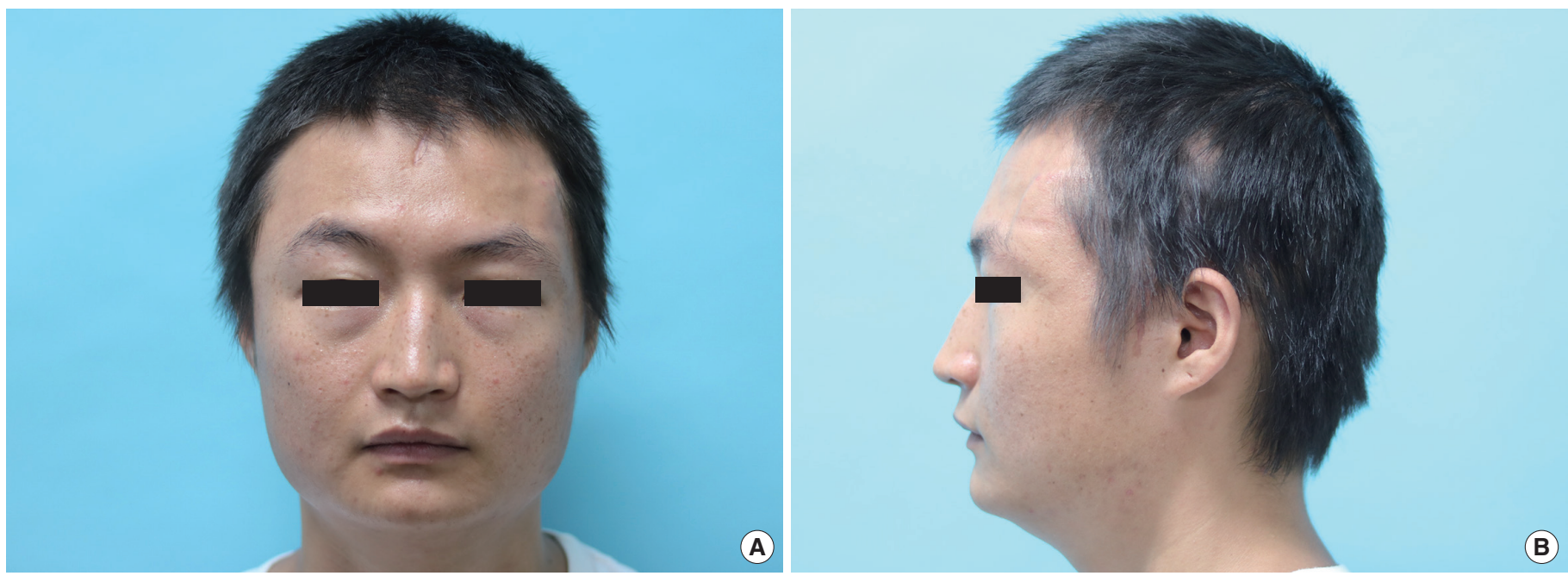

Fig. 4. Postoperative results at 3 months after surgery. There was no implant displacement, seroma formation, or wound infection. Note the symmetric contour of the temporal region; (A) frontal view and (B) lateral view.

perficial temporal artery was not palpable in the temporal area due to the injury in the previous operation. Moreover, asymmetry may have been a consequence of malpositioning of the temporalis muscle at the end of the previous operation, resulting in atrophy of the entire muscle. The frontalis muscle, periorbital muscle motion, and the temporal extension of the buccal fat pad were absent, and there was a bony defect; consequently, the temporal area was almost empty.

Cranioplasty is performed to provide protective coverage to the brain and to restore a pleasing aesthetic contour to the cranium [5]. In this case, temporal hollowing was caused by a combination of a temporal bone defect, muscle atrophy, and atrophy of the superficial temporal fat pad.

Solutions for protective coverage of the cranium include an autogenous graft and the insertion of alloplastic implants [5]. Autologous bone flaps, frozen or banked, deriving from the subcutaneous tissue of the abdomen, remain the most common source.

However, an autologous bone graft could not be performed in this patient, for multiple reasons. First, if a bone graft is performed, grafting should be performed by harvesting the contralateral parietal bone. The patient wanted no further scars to be left on the scalp and did not wish to undergo major surgery that would take a long time. Second, the patient did not want donor site morbidity due to bone harvesting at the rib or another site. Third, the viability of the grafted bone was expected to be poor due to the absence of periosteum at the site of the bony defect.

The authors decided to use titanium mesh as an alternative to the bone graft. Titanium mesh has the following advantages over bone graft. First, in this case, since the titanium mesh was customized and prepared in a prefabricated form, it was possible to identify the shape of the bony defect precisely and to fabricate a titanium mesh model with the corresponding shape and size. During sur- gery, using a customized craniofacial implant allows surgeons to overcome intraoperative difficulties in achieving ideal form and consistency [5]. Second, it has better plasticity and mechanical properties than bone, and can be easily fixed to the adjacent bone. Third, in specific facial regions where the implant encroaches on the bony structure, silicone may cause osseous erosion. However, when an implant made of silicone or another material is placed on titanium, unlike bone, there is no possibility of erosion or undersurface deformities. At the same time, since the skull-titanium complex is not deformed, contours can be maintained without affecting the gross appearance. Fourth, titanium is a biocompatible material, but unlike bone, there is no need to worry about viability. Fifth, as repeated trauma and exposure of the implant may occur, it would be expected that the underlying titanium mesh support would provide protection to the brain even if the overlying implant is ruptured or removed.

Titanium mesh was used in the case described herein for temporal hollowing reconstruction with good results. A silicone implant was used for soft tissue augmentation of muscle and to address temporal fat pad atrophy. A silicone implant was used for soft tissue augmentation in this case for multiple reasons. First, stable skin coverage with a sufficiently thick skin flap is an essential prerequisite for any type of cranioplasty technique, particularly when alloplastic materials are used [6]. In this case, in the area of the bony defect, the skin flap adhered to the dura. Fortunately, after dissection of the skin flap from the dura, the thickness of the skin flap was sufficiently thick to insert the alloplastic material. It was also expected that closure would be possible without problems such as extrusion or tension even if a silicone implant was inserted. Second, a silicone implant provides a reliably soft texture and can be easily trimmed in the operating room. Even if silicone is customized, it is necessary to cover the skin flap and to shave it ac- 
cording to its shape and thickness. Therefore, the author prepared a silicone block and performed intraoperative shaving [6]. Third, because the patient requested reconstruction with a natural, soft, yet strong material, the soft tissue was augmented using a silicone implant, which is soft but resistant to deforming forces without fractures. Fourth, since the patient was young, it was necessary to perform the reconstruction with a long-term non-reactive implant. Silicone was suitable as an implant material in this case because it does not undergo remodeling or resorption, and there is an absence of chemical changes, toxicity, and carcinogenicity [6]. Fifth, silicone is used to cover titanium mesh, which is a relatively hard material, because the possibility of implant exposure is higher when using only titanium mesh. Polymethylmethacrylate (PMMA) has been commonly used as a material in skull reconstruction. It provides rigidity and pleasing contours, but gives off considerable heat during the in situ curing process, and both the compression strength and tensile strength of PMMA are lower than those of cortical bone [7]. Therefore, PMMA was not suitable for skull reconstruction in this patient. There were some limitations to the treatment of this patient. Silicone may be useful for the reasons described above, but complications such as seroma, infection, and capsular contracture may occur, which may result in late seroma or late infection even after 1 to 2 years. Therefore, this patient requires long-term follow-up, and it would be ideal to conduct a statistical analysis of this treatment strategy through a study of multiple cases. In addition, in the process of insetting a silicone implant, silicone movement can occur, although in this case the silicone implant was fixed to the titanium mesh and the surrounding galea, which is a relatively fixed structure. Because the fixation is not rigid, malposition and capsular contracture can occur, requiring long-term follow-up. To improve the asymmetry caused by the defect of the upper face, a titanium mesh was used for the bone defect, and silicone was used for the soft tissue defect to improve asymmetry. Reconstruction of the temporal hollowing using a titanium mesh and silicone implant is simple, quick, and allows the defect to be resolved in a single operation. No case has been reported involving reconstruction simultaneously using the alloplastic materials titanium and silicone. Moreover, the results of the procedure provided great satisfaction to the patient, not only from an aesthetic standpoint but also due to the restoration of a functional barrier resistant to potential trauma.

\section{CONFLICT OF INTEREST}

No potential conflict of interest relevant to this article was reported.

\section{ETHICAL APPROVAL}

The study was performed in accordance with the principles of the Declaration of Helsinki.

\section{PATIENT CONSENT}

The patient provided written informed consent for the publication and the use of his images.

\section{ORCID}

Han Byeol Jin (https://orcid.org/0000-0002-0965-4372) Jee Hyeok Chung (https://orcid.org/0000-0003-2782-8898)

Kyung Sik Kim (https://orcid.org/0000-0002-2101-9654)

Seung Hong Kim (https://orcid.org/0000-0001-8092-8551)

Joon Choe (https://orcid.org/0000-0002-0860-3231)

Jeong Yeol Yang (https://orcid.org/0000-0002-4343-4429)

\section{REFERENCES}

1. Lacey M, Antonyshyn O, MacGregor JH. Temporal contour deformity after coronal flap elevation: an anatomical study. J Craniofac Surg 1994;5:223-7.

2. Kadri PA, Al-Mefty O. The anatomical basis for surgical preservation of temporal muscle. J Neurosurg 2004;100:517-22.

3. Kim S, Matic DB. The anatomy of temporal hollowing: the superficial temporal fat pad. J Craniofac Surg 2005;16:651-4.

4. Matic DB, Kim S. Temporal hollowing following coronal incision: a prospective, randomized, controlled trial. Plast Reconstr Surg 2008; 121:379e-385e.

5. Zhong S, Huang GJ, Susarla SM, et al. Quantitative analysis of dualpurpose, patient-specific craniofacial implants for correction of temporal deformity. Neurosurgery 2015;11 Suppl 2:220-9.

6. Habal MB, Chalian VA. Experience with prefabricated silicone implants for reconstruction in facially deformed patients. J Prosthet Dent 1974;32:292-9.

7. Chicarilli ZN, Ariyan S. Cranioplasty with a silicone prosthesis and split rib grafts. Head Neck Surg 1986;8:355-62. 